\title{
Propionibacterium cyclohexanicum sp. nov., a New Acid-Tolerant $\omega$-Cyclohexyl Fatty Acid-Containing Propionibacterium Isolated from Spoiled Orange Juice
}

\author{
KAORI KUSANO,${ }^{1 *}$ HIDEKO YAMADA,${ }^{1}$ MOTOHIRO NIWA, ${ }^{1}$ AND KAZUHIDE YAMASATO ${ }^{2}$ \\ $R \& D$ Office, KIRIN Beverage Corporation, Samukawa-machi, Koza-gun, Kanagawa Prefecture 253-01, ${ }^{1}$ and Culture \\ Collection Center, Tokyo University of Agriculture, Setagaya-ku, Tokyo $156,^{2}$ Japan
}

\begin{abstract}
A non-spore-forming, coryneform bacterium, strain $\mathrm{TA}-12^{\mathrm{T}}$, was isolated from spoiled off-flavor orange juice. Growth of this organism occurs at pH 3.2 to 7.5, and optimum growth occurs at pH values between 5.5 and 6.5. This organism produces lactic acid, propionic acid, and acetic acid from glucose. It is catalase negative. The cells are heat resistant and can withstand a temperature of $90^{\circ} \mathrm{C}$ for $10 \mathrm{~min}$. The DNA G+C content is 66.8 mol\%. This strain has an $\mathrm{MK}-9\left(\mathrm{H}_{4}\right)$ respiratory quinone system and contains meso-diaminopimelic acid in its cell wall, and $\omega$-cyclohexyl undecanoic acid is the major cellular fatty acid. The results of a phylogenetic analysis of the 16S rRNA gene of this organism indicated that its highest level of homology is its level of homology with the representative of the classical propionibacteria, Propionibacterium freudenreichii $(\mathbf{9 7 . 1 \% )}$. Strain TA-12 ${ }^{\mathbf{T}}$ is phenotypically similar to $P$. freudenreichii, but it produces a large amount of lactic acid and has a distinct fatty acid composition, acid tolerance, and heat resistance, which differentiate it from $P$. freudenreichii and other propionic acid-producing bacteria. On the basis of these findings we propose the name Propionibacterium cyclohexanicum sp. nov. for this organism. The type strain is TA-12 (= IAM $14535=$ NRIC 0247).
\end{abstract}

Microbial contamination and growth in consumer products is one of the major problems in the food industry. It has been generally recognized that the major microbial contaminants in the fruit juice industry are yeasts, molds, and lactic acid bacteria, as these organisms prefer or can tolerate the low $\mathrm{pH}$ values of fruit juices. The growth of contaminant bacteria other than lactic acid bacteria in packaged juices was not reported until about 10 years ago. In 1984, spore-forming bacteria which can germinate and grow in apple juice were found by Cerny et al. (2), and similar spore formers were isolated from fruit juices in 1994 (26). The organism isolated by Cerny et al. was named Bacillus acidoterrestris by Deinhard et al. (8) and was reclassified as Alicyclobacillus acidoterrestris by Wisotzkey et al. (35) in 1992. The genus Alicyclobacillus is acidophilic and either thermophilic or thermotolerant, and characteristically the members of this genus contain $\omega$-alicylic fatty acid.

In 1993, we isolated a coryneform bacterium, strain TA-12 ${ }^{\mathrm{T}}$, from spoiled orange juice. Like members of the genus Alicyclobacillus, this organism is acid tolerant and $\omega$-cyclohexyl fatty acid is its major cellular fatty acid, but it does not form spores.

In this paper we present a taxonomic characterization of this bacterium and propose the name Propionibacterium cyclohexanicum for it.

\section{MATERIALS AND METHODS}

Bacterial strains. Strain TA-12 $2^{\mathrm{T}}$ was isolated from spoiled off-flavor orange juice by using $\mathrm{pH}$ 7.0 Standard Method Agar ("Nissui" medium; Nissui Pharmaceutical Co., Ltd., Tokyo, Japan) incubated at $35^{\circ} \mathrm{C}$. This medium contained (per liter) $5 \mathrm{~g}$ of peptone, $2.5 \mathrm{~g}$ of yeast extract, $1 \mathrm{~g}$ of glucose, and $15 \mathrm{~g}$ of agar. Propionibacterium freudenreichii ATCC $6207^{\mathrm{T}}$ was used as a reference strain Strain TA-12 ${ }^{\mathrm{T}}$ has been deposited in the IAM Culture Collection at the Institute of Molecular and Cellular Biosciences (formerly Institute of Applied Microbiology), University of Tokyo, as strain IAM $14535^{\mathrm{T}}$ and in the Culture Collection Center, Tokyo University of Agriculture, as strain NRIC $0247^{\mathrm{T}}$.

Media and cultivation. Strain TA-12 $2^{\mathrm{T}}$ was cultivated in PYG medium containing (per liter) $5 \mathrm{~g}$ of peptone (Difco Laboratories, Detroit, Mich.), $2.5 \mathrm{~g}$ of

* Corresponding author. Fax: 81-467-74-6025. E-mail: k-kusano @kirin.co.jp..beverage.co.jp. yeast extract (Difco Laboratories), and $5 \mathrm{~g}$ of glucose. The $\mathrm{pH}$ was adjusted to 6.5 by adding sulfuric acid prior to autoclaving. For solid medium, $1.5 \%$ agar was added. Strain TA-12 ${ }^{\mathrm{T}}$ and Propionibacterium freudenreichii ATCC $6207^{\mathrm{T}}$ were grown in stationary cultures at 35 and $30^{\circ} \mathrm{C}$, respectively. For chemotaxonomic studies strains were cultured in medium containing (per liter) $5 \mathrm{~g}$ of peptone $2.5 \mathrm{~g}$ of yeast extract, and $1 \mathrm{~g}$ of glucose $(\mathrm{pH} 6.5)$. The optimal temperature and optimal $\mathrm{pH}$ were determined by measuring the optical density at $580 \mathrm{~nm}$.

Morphological features. Gram staining was carried out by the modified Hucker method (11). Spore staining was carried out by the modified method of Schaeffer and Fulton (25).

Physiological and biochemical features. Anaerobic growth was tested by using an Anaeropouch (Mitsubishi Gas Chemical Co., Inc., Tokyo, Japan). Oxidase production was tested by using a cytochrome oxidase test strip (Nissui Pharmaceutical Co., Ltd.). Other tests were conducted by using the methods described by Cowan and Steel (5). Assimilation of carbon compounds was determined by observing acid production with an API 50CH kit (BioMerieux S.A., Marcy l'Etoile, France) by the method of Deinhard et al. (8), with some modifications. To minimize ambiguous observations, low concentrations of peptone were used in the basal medium $\left(0.01 \%\right.$ peptone for strain TA $-12^{\mathrm{T}}$ and $0.05 \%$ peptone for Propionibacterium freudenreichii ATCC $6207^{\mathrm{T}}$ ). The indicator used was bromocresol purple. When the results were ambiguous, we retested without the indicator by measuring the decrease in $\mathrm{pH}$ after 1 week of incubation. When the $\mathrm{pH}$ was less than 3.8, a sugar test was considered positive. Assimilation of acetic, lactic, malic, citric, and succinic acids was determined by culturing cells in test tubes containing PYG medium without glucose and measuring the amount of growth.

Heat resistance. The heat resistance of cells was determined by the screw-cap tube technique described by Kooiman (16). A $0.1-\mathrm{ml}$ portion of a stationaryphase PYG broth culture, whose cell concentration was $10^{10}$ cells $/ \mathrm{ml}$, was in jected into $10 \mathrm{ml}$ of preheated fresh medium in a screw-cap test tube. Test tubes were preheated at various temperatures and were maintained at these temperatures for various periods of time. Cell viability was determined by examining the growth exhibited by the heated tube cultures.

Fermentation products. The fermentation products were analyzed by highperformance liquid chromatography (HPLC). Strain TA-12 ${ }^{\mathrm{T}}$ was cultured in Trypticase-yeast extract-glucose broth $(\mathrm{pH} 7.0)(6)$ at $35^{\circ} \mathrm{C}$ for 4 days. After the cells were removed by filtration through a $0.2-\mu \mathrm{m}$-pore-size membrane filter, the broth was used in the analysis. The HPLC system used was a 9000 series (Japan Spectroscopic Co., Ltd., Hachioji, Japan) equipped with a Shodex KC-811 column (Showa Denko K. K., Tokyo, Japan). The samples were eluted at $50^{\circ} \mathrm{C}$ with $3 \mathrm{mM}$ perchloric acid $(1.0 \mathrm{ml} / \mathrm{min})$ and reaction buffer $(1.5 \mathrm{ml} / \mathrm{min})$ containing $0.2 \mathrm{mM}$ bromothymol blue, $15 \mathrm{mM} \mathrm{Na}_{2} \mathrm{HPO}_{4}$, and $2 \mathrm{mM} \mathrm{NaOH}$. Organic acids were detected by $A_{445}$.

Cell wall composition. Peptidoglycan was prepared as follows. About $1 \mathrm{~g}$ of wet cells was resuspended in $10 \mathrm{ml}$ of $0.05 \mathrm{M}$ phosphate buffer $(\mathrm{pH} \mathrm{7.2)}$ and sonicated with a Handy Sonic instrument (model UR-20D; Tomy Seiko Co., Ltd., Tokyo, Japan) for $20 \mathrm{~min}$ at power level 10 . Then the sample was centri- 
fuged at $5,000 \times g$ for $10 \mathrm{~min}$. The supernatant was boiled with $4 \%$ sodium dodecyl sulfate for $40 \mathrm{~min}$ and ultracentrifuged at $100,000 \times g$ for $30 \mathrm{~min}$. The precipitated proteins were digested with a solution containing $200 \mu \mathrm{g}$ of pronase E (Sigma Chemical Co., St. Louis, Mo.) per $\mathrm{ml}, 3 \mathrm{mg}$ of trypsin (Wako Pure Chemical Industries, Ltd., Osaka, Japan) per $\mathrm{ml}$, and $3 \mathrm{mg}$ of pepsin (Sigma Chemical Co.) per ml, and the lipids were removed by a series of ether-ethanol (1:1), chloroform-methanol (2:1), chloroform, and acetone extractions. The DNA and RNA were digested with solutions containing $40 \mu \mathrm{g}$ of DNase (Boehringer Mannheim $\mathrm{GmbH}$, Mannheim, Germany) per $\mathrm{ml}$ and $40 \mu \mathrm{g}$ of RNase (Boehringer Mannheim $\mathrm{GmbH}$ ) per $\mathrm{ml}$, respectively. The sample was finally purified by trichloroacetic acid treatment (12). The amino acid composition of the purified peptidoglycan was determined by the method described by Cohen and Michaud (4). The diaminopimelic acid isomers were analyzed by the method described by Staneck and Roberts (28). The glycolyl residue was determined by the method of Uchida and Aida (34).

Whole-cell sugar pattern. Cell sugars were analyzed by the method described by Staneck and Roberts (28).

Respiratory quinone system. Menaquinones were extracted and analyzed by the method described by Tamaoka et al. (31).

Fatty acid analysis. Cell lipids were prepared from about $1 \mathrm{~g}$ of wet cells by the Bligh-Dyer method (1). The cells were broken with a Waring blender containing glass beads ( 80 to 100 mesh), and the lipids were extracted with a chloroformmethanol mixture $(2: 1)$. The extracted lipids were hydrolyzed at $60^{\circ} \mathrm{C}$ for $5 \mathrm{~min}$ with $0.5 \mathrm{~N} \mathrm{NaOH}$ dissolved in methanol and then methylated at $60^{\circ} \mathrm{C}$ for $5 \mathrm{~min}$ with methanol containing $7 \%$ boron trifluoride (19). Gas chromatography-mass spectrometry was performed with a model 5971A mass spectrometer (HewlettPackard GmbH, Waldboron, Germany) and a model 5890 gas chromatograph (Hewlett-Packard $\mathrm{GmbH}$ ) equipped with a type DB-23 capillary column (J\&B Scientific, Inc., Folsom, Calif.). The column temperature was increased from 130 to $210^{\circ} \mathrm{C}$ at a rate of $3^{\circ} \mathrm{C} / \mathrm{min}$.

Extraction and purification of DNA. About $1 \mathrm{~g}$ of wet cells was resuspended in $15 \mathrm{ml}$ of TE buffer (10 mM Tris, $1 \mathrm{mM}$ EDTA; $\mathrm{pH} 8.0$ ), and $5 \mathrm{mg}$ of lysozyme (Wako Pure Chemical Industries, Ltd.), 2,000 U of achromopeptidase (Wako Pure Chemical Industries, Ltd.), and $30 \mu$ l of $5 \mathrm{M} \mathrm{NaCl}$ (final concentration, 0.01 M) were added. This mixture was incubated at $37^{\circ} \mathrm{C}$ for $1 \mathrm{~h}$ and then at $70^{\circ} \mathrm{C}$ for $10 \mathrm{~min}$. Total lysis was achieved by adding $375 \mu \mathrm{l}$ of $20 \%$ sodium dodecyl sulfate. Proteins were digested by overnight incubation at $55^{\circ} \mathrm{C}$ with $1.5 \mathrm{mg}$ of proteinase K (Wako Pure Chemical Industries, Ltd.). The residual proteins and polysac charides were removed by hexadecyltrimetylammonium bromide treatment (18) Further purification was carried out by repeated incubation at $55^{\circ} \mathrm{C}$ with proteinase $\mathrm{K}$, deproteinization with phenol and chloroform-isoamyl alcohol (24:1) and precipitation with isopropyl alcohol.

Measurement of the $\mathbf{G}+\mathbf{C}$ content of the DNA. Purified DNAs were hydrolyzed into nucleosides and analyzed by HPLC by the method of Tamaoka and Komagata (30), with some modifications. The heat-denatured DNA solution was incubated at $50^{\circ} \mathrm{C}$ overnight with nuclease P1 (Yamasa Shoyu Co., Ltd., Choshi, Japan). The HPLC system used was a 9000 series (Japan Spectroscopic Co., Ltd.) equipped with a CrestPak C18T-5 column (Japan Spectroscopic Co., Ltd.), and the nucleosides were eluted with $10 \mathrm{mM} \mathrm{KH}_{2} \mathrm{PO}_{4}(\mathrm{pH} \mathrm{3.5)}$

Determination of the $16 \mathrm{~S}$ rRNA gene sequence and phylogenetic analysis. The $16 \mathrm{~S}$ rRNA gene was amplified in vitro by PCR (23) by using a 16S rRNA gene-specific primer pair consisting of 5'-TTGGATCCAGAGTTTGATCCTG GCTCAG-OH-3' (positions 8 to 27; Escherichia coli numbering system [9]), which contains a BamHI site, and 5'-TTAAGCTTAAGGAGGTGATCCAGC CGCA-OH-3' (positions 1521 to $1540 ;$ E. coli numbering system), which contains a HindIII site. Each cycle consisted of $1 \mathrm{~min}$ of denaturation at $94^{\circ} \mathrm{C}, 2 \mathrm{~min}$ of annealing at $55^{\circ} \mathrm{C}$, and $3 \mathrm{~min}$ of extension at $72^{\circ} \mathrm{C}$. A total of 30 cycles were followed by rapid cooling to $4^{\circ} \mathrm{C}$. The amplified fragments were digested with Bam HI and HindIII. The digests were checked for length by $1 \%$ agarose gel electrophoresis and were purified by extraction with a Geneclean II kit (Bio 101, Inc., La Jolla, Calif.). The purified DNA fragments were ligated with plasmid vector pUC119 which had been double digested with BamHI and HindIII by using a DNA ligation kit (Takara Shuzo Co., Ltd., Kyoto, Japan). Subcloning was carried out by digestion with restriction enzymes EcoRI, SalI, and SphI. The cloned DNAs were purified by polyethylene glycol precipitation (33). The purified plasmids were sequenced by using a Taq DyeDeoxy terminator cycle sequencing kit (Perkin-Elmer Co., Norwalk, Conn.) and were analyzed with a model ABI 373A DNA sequencer (Perkin-Elmer Co.). The primary sequences of the TA-12 $16 \mathrm{~S}$ rRNA gene were generated by aligning and combining partial sequences by using DNASIS software (Hitachi Software Engineering Co., Ltd., Yokohama, Japan). The DNA sequences which ranged from positions 261 to 1455 ( $E$. coli numbering system) were aligned on the basis of the secondary structure of the $16 \mathrm{~S}$ rRNA. Positions at which the bases were uncertain and at which gaps were generated by the comparative alignment were omitted from the analysis. A total of 806 nucleotides were used. A phylogenetic tree was constructed by using Clustal W software (32).

Nucleotide sequence accession numbers. The sequence of Propionibacterium cyclohexanicum TA-12 ${ }^{\mathrm{T}}$ (accession no. D82046) was aligned with previously published sequences obtained from the EMBL, GenBank, and DDBJ databases under the following accession numbers: Aeromicrobium erythreus, M37200; Arthrobacter globiformis DSM 20124 ${ }^{\mathrm{T}}$, M23411; Luteococcus japonicus IFO $12422^{\mathrm{T}}$, D21245; Micrococcus luteus ATCC 381, M38242; Nocardioides albus DSM
43109 ${ }^{\mathrm{T}}, \mathrm{X53211}$; Propionibacterium acidipropionici DSM 20272, X53221; Propionibacterium acnes DSM $1897^{\mathrm{T}}, \mathrm{X} 53218$; Propionibacterium freudenteichii DSM $20271^{\mathrm{T}}$, X53217; Propionibacterium jensenii DSM 20535 ${ }^{\mathrm{T}}, \mathrm{X} 53219$; Propionibacterium propionicus DSM $43307^{\mathrm{T}}, \mathrm{X} 53216$; Propionibacterium thoenii DSM $20276^{\mathrm{T}}$, X53220; Terrabacter tumescens NCIB $8914^{\mathrm{T}}$, X53215; and Bacillus subtilis, X00007. The sequence of the $16 \mathrm{~S}$ ribosomal DNA gene of Propioniferax innocua NCTC $11082^{\mathrm{T}}$ was obtained from reference 20 .

\section{RESULTS}

Morphological features. Strain TA $-12^{\mathrm{T}}$ was a gram-positive, nonmotile, pleomorphic, rod-shaped bacterium whose cells were 1.5 to $3.0 \mu \mathrm{m}$ long and 1.1 to $1.6 \mu \mathrm{m}$ wide. The cells were club shaped and bent, and some swellings were observed. Spores were not observed. The colonies on the surfaces of PYG agar plates after 3 days of anaerobic growth were circular, white to creamy, translucent, and 0.2 to $0.5 \mathrm{~mm}$ in diameter.

Physiological and biochemical features. Lactic acid, propionic acid, and acetic acid were formed as a result of fermentation at a molar ratio of 5:4:2, respectively. The physiological, biochemical, and chemotaxonomic characteristics of strain TA$12^{\mathrm{T}}$ and Propionibacterium species are shown in Table 1 . Some data for Propionibacterium freudenreichii ATCC $6207^{\mathrm{T}}$ obtained in this study are included in Table 1. Propionibacterium freudenreichii ATCC $6207^{\mathrm{T}}$ was catalase positive, but strain $\mathrm{TA}-12^{\mathrm{T}}$ was catalase negative. Strain TA-12 ${ }^{\mathrm{T}}$ was aerotolerant, forming colonies on the surface of agar plate medium incubated aerobically. When cells were suspended and grown in semisolid medium, growth occurred evenly throughout the medium except for the surface layer, where it was less vigorous. Both strain TA- $12^{\mathrm{T}}$ and strain ATCC $6207^{\mathrm{T}}$ grew at 20 to $40^{\circ} \mathrm{C}$, and the optimum growth temperatures were $35^{\circ} \mathrm{C}$ for strain $\mathrm{TA}-12^{\mathrm{T}}$ and 30 to $32^{\circ} \mathrm{C}$ for Propionibacterium freudenreichii. Strain TA- $12^{\mathrm{T}}$ grew at $\mathrm{pH} 3.2$ to 7.5 , and the optimum $\mathrm{pH}$ was 5.5 to 6.5. Strain ATCC $6207^{\mathrm{T}}$ grew at $\mathrm{pH} 4.5$ to 8.5 , and optimum growth occurred at $\mathrm{pH} 6.5$ to 7.0 . Strain TA- $12^{\mathrm{T}}$ was resistant to heating at $90^{\circ} \mathrm{C}$ for $10 \mathrm{~min}$. The comparative carbon compound assimilation profiles of Propionibacterium species are shown in Table 2 . Strain TA $-12^{\mathrm{T}}$ could assimilate 23 of the 54 carbon compounds tested, including glycerol, L-xylose, galactose, D-glucose, D-fructose, D-mannose, amygdalin, arbutin, esculin, salicin, cellobiose, maltose, lactose, saccharose, trehalose, melezitose, D-turanose, D-fucose, acetic acid, lactic acid, malic acid, citric acid, and succinic acid, whereas Propionibacterium freudenreichii ATCC $6207^{\mathrm{T}}$ assimilated 14 carbon compounds, including glycerol, erythritol, ribose, adonitol, galactose, D-glucose, D-fructose, D-mannose, inositol, acetic acid, lactic acid, malic acid, citric acid, and succinic acid.

Chemotaxonomic features. The major menaquinone of strain TA-12 ${ }^{\mathrm{T}}$ was MK-9 $\left(\mathrm{H}_{4}\right)$ and the $\mathrm{G}+\mathrm{C}$ content was 66.8 mol\%. The major cellular fatty acid was $\omega$-cyclohexyl undecanoic acid, which accounted for $52.7 \%$ of the total fatty acids. The straight-chain and anteiso-branched fatty acids included $16.8 \% n-\mathrm{C}_{15}, 6.4 \%$ anteiso- $\mathrm{C}_{15}, 2.8 \% n-\mathrm{C}_{16}$, and $5.3 \% n-\mathrm{C}_{17}$, and the other fatty acids were not determined. The cell wall peptidoglycan contained meso-diaminopimelic acid, glutamic acid, and alanine at a molar ratio of 1:1:2. The whole-cell sugars were galactose, mannose, glucose, ribose, and rhamnose. An unidentified sugar spot was observed; the $R_{f}$ value of this sugar was 0.38 , which is almost the $R_{f}$ value of fucose on a chromatogram.

Phylogenetic analysis. Table 3 shows the 16S rRNA gene similarity values for strain $\mathrm{TA}-12^{\mathrm{T}}$ and allied gram-positive bacteria with high $\mathrm{G}+\mathrm{C}$ contents. The level of similarity between strain TA-12 ${ }^{\mathrm{T}}$ and Propionibacterium freudenreichii DSM $20271^{\mathrm{T}}$ was $97.1 \%$. The levels of homology with other 
TABLE 1. Biochemical and chemotaxonomic characteristics of strain TA-12 ${ }^{\mathrm{T}}$ and Propionibacterium spp.

\begin{tabular}{|c|c|c|c|c|c|c|c|c|c|c|}
\hline Characteristic & Strain TA-12 ${ }^{\mathrm{T}}$ & $\begin{array}{l}\text { Propionibacterium } \\
\text { freudenreichii }^{a}\end{array}$ & $\begin{array}{l}\text { Propionibacterium } \\
\text { jensenii }^{a}\end{array}$ & $\begin{array}{l}\text { Propionibacterium } \\
\text { thoeniil }^{a}\end{array}$ & $\begin{array}{l}\text { Propionibacterium } \\
\text { acidipropionici }^{a}\end{array}$ & $\begin{array}{l}\text { Propionibacterium } \\
\text { acnes }^{a}\end{array}$ & $\begin{array}{l}\text { Propionibacterium } \\
\text { avidum }^{a}\end{array}$ & $\begin{array}{l}\text { Propionibacterium } \\
\text { granulosum }^{a}\end{array}$ & $\begin{array}{l}\text { Propionibacterium } \\
\text { lymphophilum }^{a}\end{array}$ & $\begin{array}{l}\text { Propionibacterium } \\
\text { propionicus }^{b}\end{array}$ \\
\hline Catalase & $-^{c}$ & + & $d+$ & + & $d+$ & $d+$ & + & + & $d+$ & - \\
\hline Relationship to oxygen & Aerotolerant & $\begin{array}{l}\text { Anaerobic to } \\
\text { aerotolerant }\end{array}$ & $\begin{array}{l}\text { Anaerobic to } \\
\text { aerotolerant }\end{array}$ & $\begin{array}{l}\text { Less strictly } \\
\text { anaerobic }\end{array}$ & $\begin{array}{l}\text { Anaerobic to } \\
\text { aerotolerant }\end{array}$ & $\begin{array}{l}\text { Anaerobic to } \\
\text { aerotolerant }\end{array}$ & $\begin{array}{l}\text { Anaerobic or } \\
\text { microaerophilic }\end{array}$ & $\begin{array}{l}\text { Anaerobic or } \\
\text { ic microaerophilic }\end{array}$ & Anaerobic & Facultatively anaerobic \\
\hline Hydrolysis of gelatin & - & - & - & - & - & + & + & $d-$ & $d+$ & $\mathrm{d}$ \\
\hline Hydrolysis of starch & - & - & - & $d+$ & - & - & - & - & $\mathrm{d}-$ & $\mathrm{d}$ \\
\hline Hydrolysis of esculin & + & + & + & + & + & - & + & - & - & - \\
\hline Hydrolysis of urea & + & $-{ }^{d}$ & $\mathrm{ND}^{e}$ & ND & ND & ND & ND & ND & ND & - \\
\hline $\begin{array}{l}\text { Reduction of nitrate to } \\
\text { nitrite }\end{array}$ & - & $\mathrm{d}$ & - & - & + & $d+$ & - & - & d & + \\
\hline Formation of indole & - & - & - & - & - & $d+$ & - & - & - & - \\
\hline $\mathrm{pH}$ range for growth & $3.2-7.5$ & $4.5-8.5^{d}$ & ND & ND & ND & ND & ND & ND & ND & ND \\
\hline $\begin{array}{l}\text { Optimum temp for } \\
\text { growth }\left({ }^{\circ} \mathrm{C}\right)\end{array}$ & 35 & $30-32$ & $30-32$ & $30-32$ & $30-32$ & $36-37$ & $36-37$ & $36-37$ & $36-37$ & $35-37$ \\
\hline Fermentation products I & $\begin{array}{l}\text { Lactic acid, } \\
\text { propionic } \\
\text { acid, acetic } \\
\text { acid }\end{array}$ & $\begin{array}{l}\text { Propionic acid, } \\
\text { acetic acid }\end{array}$ & $\begin{array}{l}\text { Propionic acid, } \\
\text { acetic acid }\end{array}$ & $\begin{array}{l}\text { Propionic acid, } \\
\text { acetic acid }\end{array}$ & $\begin{array}{l}\text { Propionic acid, } \\
\text { acetic acid }\end{array}$ & $\begin{array}{l}\text { Propionic acid, } \\
\text { acetic acid }\end{array}$ & $\begin{array}{l}\text { Propionic acid, } \\
\text { acetic acid }\end{array}$ & $\begin{array}{l}\text { Propionic acid, } \\
\text { acetic acid }\end{array}$ & $\begin{array}{l}\text { Propionic acid, } \\
\text { acetic acid }\end{array}$ & $\begin{array}{l}\text { Acetic acid, propionic } \\
\text { acid, lactic acid, } \\
\text { succinic acid }\end{array}$ \\
\hline $\begin{array}{l}\text { Major respiratory } \\
\text { quinone }\end{array}$ & MK-9( $\left.\mathrm{H}_{4}\right)$ & MK-9 $\left(\mathrm{H}_{4}\right)^{f}$ & MK-9 $\left(\mathrm{H}_{4}\right)^{f}$ & MK-9( $\left.\mathrm{H}_{4}\right)^{f}$ & MK-9 $\left(\mathrm{H}_{4}\right)^{f}$ & MK-9( $\left.\mathbf{H}_{4}\right)^{f}$ & MK-9 $\left(\mathrm{H}_{4}\right)^{f}$ & MK-9 $\left(\mathrm{H}_{4}\right)^{f}$ & MK-9 $\left(\mathrm{H}_{4}\right)^{f}$ & MK-9 $\left(\mathrm{H}_{4}\right)$ \\
\hline $\mathrm{G}+\mathrm{C}$ content $(\mathrm{mol} \%)$ & 66.8 & $64-67$ & $65-68$ & $66-67$ & $66-68$ & $57-60$ & $62-63$ & $61-63$ & $53-54$ & $63-65$ \\
\hline $\begin{array}{l}\text { Isomer of } A_{2} \mathrm{pm} \text { in cell } r \\
\text { wallg }\end{array}$ & meso- $\mathrm{A}_{2} \mathrm{pm}$ & meso- $\mathrm{A}_{2} \mathrm{pm}$ & LL- $\mathrm{A}_{2} \mathrm{pm}$ & $\mathrm{LL}-\mathrm{A}_{2} \mathrm{pm}$ & $\mathrm{LL}-\mathrm{A}_{2} \mathrm{pm}$ & $\mathrm{LL}-(m e s o)-\mathrm{A}_{2} \mathrm{pm}$ & $\mathrm{LL}-(m e s o)-\mathrm{A}_{2} \mathrm{pm}$ & $\mathrm{LL}-\mathrm{A}_{2} \mathrm{pm}$ & & $\mathrm{LL}-\mathrm{A}_{2} \mathrm{pm}$ \\
\hline Major fatty acid & $\omega$-Cyclohexane & Branched & ND & ND & Branched & Branched & Branched & Branched & Branched & Straight \\
\hline $\begin{array}{l}\text { Sugars in } \\
\text { polysaccharide }{ }^{h}\end{array}$ & $\begin{array}{l}\text { Glucose, } \\
\text { galactose, } \\
\text { mannose, } \\
\text { rhamnose, } \\
\text { ribose }\end{array}$ & $\begin{array}{l}\text { Galactose, } \\
\text { mannose, } \\
\text { rhamnose }\end{array}$ & $\begin{array}{l}\text { Glucose, } \\
\text { galactose, } \\
\text { mannose }\end{array}$ & $\begin{array}{l}\text { Glucose, } \\
\text { galactose }\end{array}$ & $\begin{array}{l}\text { Galactose, } \\
\text { glucose, } \\
\text { mannose }\end{array}$ & $\begin{array}{l}\text { Glucose, } \\
\text { mannose, } \\
\text { (galactose) }\end{array}$ & $\begin{array}{l}\text { Glucose, } \\
\text { mannose, } \\
\text { (galactose) }\end{array}$ & $\begin{array}{l}\text { Galactose, } \\
\text { mannose }\end{array}$ & $\begin{array}{l}\text { Galactose, } \\
\text { glucose } \\
\text { mannose }\end{array}$ & $\begin{array}{l}\text { Glucose, galactose, } \\
\text { mannose }\end{array}$ \\
\hline $\begin{array}{l}\text { Amino acids in cell } \\
\text { wall }^{g}\end{array}$ & $\begin{array}{l}\text { Alanine, } \\
\text { glutamic acid, } \\
\text { meso- } \mathrm{A}_{2} \mathrm{pm}\end{array}$ & $\begin{array}{l}\text { Alanine, } \\
\text { glutamic acid, } \\
\text { meso- } \mathrm{A}_{2} \mathrm{pm}\end{array}$ & $\begin{array}{l}\text { Alanine, } \\
\text { glutamic acid, } \\
\text { glycine, LL- } \\
\mathrm{A}_{2} \mathrm{pm}\end{array}$ & $\begin{array}{l}\text { Alanine, } \\
\text { glutamic acid, } \\
\text { glycine, LL- } \\
\mathrm{A}_{2} \mathrm{pm}\end{array}$ & $\begin{array}{l}\text { Alanine, } \\
\text { glutamic acid, } \\
\text { glycine, LL- } \\
\mathrm{A}_{2} \mathrm{pm}\end{array}$ & $\begin{array}{l}\text { Alanine, } \\
\text { glutamic acid, } \\
\text { (glycine), } \mathrm{LL}- \\
(\text { meso })-\mathrm{A}_{2} \mathrm{pm}\end{array}$ & $\begin{array}{l}\text { Alanine, } \\
\text { glutamic acid, } \\
\text { (glycine), LL- } \\
\left(\text { meso)-A } \mathrm{A}_{2} \mathrm{pm}\right.\end{array}$ & $\begin{array}{l}\text { Alanine, } \\
\text { glutamic acid, } \\
\text { glycine, LL- } \\
\mathrm{A}_{2} \mathrm{pm}\end{array}$ & $\begin{array}{l}\text { Alanine, } \\
\text { glutamic acid, } \\
\text { lysine }\end{array}$ & $\begin{array}{l}\text { Glycine, glutamic acid, } \\
\text { alanine, } \mathrm{LL}-\mathrm{A}_{2} \mathrm{pm}\end{array}$ \\
\hline
\end{tabular}

${ }^{a}$ Data from reference 7

${ }^{b}$ Data from references 3 and 24 .

$c_{-,}, 90 \%$ or more of the strains are negative;,$+ 90 \%$ or more of the strains are positive; $d, 11$ to $89 \%$ of the strains are positive; $d+, 40$ to $90 \%$ of the strains are positive; $d-, 10$ to $40 \%$ of the strains are positive. ${ }^{d}$ Data for strain ATCC $6207^{\mathrm{T}}$ determined in this study.

$\mathrm{ND}$, no data available.

Data from reference 14.

h ${ }^{2}$ Sugars in 
TABLE 2. Acid formation from various carbon sources by strain TA-12 ${ }^{\mathrm{T}}$ and Propionibacterium spp.

\begin{tabular}{|c|c|c|c|c|c|c|c|c|c|c|}
\hline \multirow{2}{*}{$\begin{array}{l}\text { Carbon } \\
\text { source }\end{array}$} & \multicolumn{10}{|c|}{ Acid formation by: } \\
\hline & $\begin{array}{l}\text { Strain } \\
\text { TA-12 }\end{array}$ & $\begin{array}{l}\text { Propionibacterium } \\
\quad \text { freudenreichiia }\end{array}$ & $\begin{array}{l}\text { Propionibacterium } \\
\text { jensenii }^{a}\end{array}$ & $\begin{array}{l}\text { Propionibacterium } \\
\text { thoenii }^{a}\end{array}$ & $\begin{array}{l}\text { Propionibacterium } \\
\text { acidipropionici }^{a}\end{array}$ & $\begin{array}{l}\text { Propionibacterium } \\
\text { acnes }^{a}\end{array}$ & $\begin{array}{l}\text { Propionibacterium } \\
\text { avidum }^{a}\end{array}$ & $\begin{array}{l}\text { Propionibacterium } \\
\text { granulosum }^{a}\end{array}$ & $\begin{array}{l}\text { Propionibacterium } \\
\text { hymphophilum }^{a}\end{array}$ & $\begin{array}{l}\text { Propionibacterium } \\
\text { propionicus }^{b}\end{array}$ \\
\hline Glycerol & $+^{c}$ & + & + & + & + & $d+$ & + & + & - & d \\
\hline Erythritol & - & + & + & $d+$ & + & $d+$ & + & - & + & d \\
\hline D-Arabinose & - &,$+-^{d}$ & - & - & + & - & $d+$ & - & - & - \\
\hline Ribose & - & $\mathrm{d}+$ & + & + & + & $d+$ & $d+$ & $\mathrm{d}-$ & + & d \\
\hline D-Xylose & - & - & $d+$ & $d+$ & $d+$ & - & $d-$ & - & - & - \\
\hline L-Xylose & + & $-{ }^{d}$ & $\mathrm{ND}^{e}$ & ND & ND & ND & ND & ND & ND & ND \\
\hline Adonitol & - & $\mathrm{d}+$ & $d+$ & $d+$ & + & $d+$ & $d+$ & - & + & $\mathrm{d}$ \\
\hline Galactose & + & + & + & + & + & $d+$ & + & $d-$ & - & d \\
\hline D-Glucose & + & + & + & + & + & $d+$ & + & + & + & + \\
\hline D-Fructose & + & + & + & + & + & $d+$ & + & + & + & + \\
\hline D-Mannose & + & + & + & + & + & $d+$ & + & + & - & d \\
\hline L-Sorbose & - & - & - & - & $d+$ & - & - & - & _- & - \\
\hline Rhamnose & - & - & - & - & + & - & - & - & - & - \\
\hline Dulcitol & - & - & - & - & - & - & - & - & - & - \\
\hline Inositol & - & $d+$ & $d+$ & $d+$ & + & $\mathrm{d}-$ & $d+$ & - & $d+$ & d \\
\hline Mannitol & - & - & + & - & + & d- & d- & $d+$ & - & + \\
\hline Sorbitol & - & - & - & $d+$ & + & $d+$ & - & - & - & d \\
\hline Amygdalin & + & - & $d+$ & $d+$ & - & - & - & $d-$ & - & $\mathrm{d}$ \\
\hline Arubtin & + & $-{ }^{d}$ & ND & ND & ND & ND & ND & ND & ND & ND \\
\hline Esculin & + & - & - & + & $d+$ & - & - & - & - & ND \\
\hline Salicin & + & - & + & $d+$ & + & - & $d+$ & - & - & d \\
\hline Cellobiose & + & - & $d-$ & - & + & - & - & - & - & - \\
\hline Maltose & + & - & $d+$ & $d+$ & + & - & + & $d+$ & + & + \\
\hline Lactose & + & $d-$ & $d+$ & d- & + & - & $d+$ & - & - & d \\
\hline Melibiose & \pm & d- & + & $d+$ & $d+$ & - & $d+$ & d- & - & d \\
\hline Saccharose & + & - & + & $d+$ & + & - & + & + & $\mathrm{d}-$ & + \\
\hline Trehalose & + & - & + & + & + & - & + & $d+$ & - & d \\
\hline Inulin & - & - & - & - & - & - & - & - & - & - \\
\hline Melezitose & + & - & $d+$ & $d+$ & + & - & $d+$ & $d-$ & - & - \\
\hline D-Raffinose & - & - & + & $d+$ & $d-$ & - & $d+$ & $d+$ & - & + \\
\hline Starch & - & - & - & + & + & - & - & - & $d+$ & $\mathrm{d}$ \\
\hline Glycogen & - & - & - & $d+$ & - & - & - & - & - & - \\
\hline D-Turanose & + & $-^{d}$ & ND & ND & ND & ND & ND & ND & ND & ND \\
\hline D-Fucose & + & $-{ }^{d}$ & ND & ND & ND & ND & ND & ND & ND & ND \\
\hline
\end{tabular}

${ }^{a}$ Data from reference 7.

${ }^{b}$ Data from references 3 and 24.

d ${ }^{d}$ Data for strain ATCC $6207^{\mathrm{T}}$ determined in this study. 
TABLE 3. $16 \mathrm{~S}$ rRNA gene sequence similarity values for strain $\mathrm{TA}-12^{\mathrm{T}}$ and related species

\begin{tabular}{|c|c|c|c|c|c|c|c|c|c|c|c|c|c|c|c|}
\hline \multirow[b]{2}{*}{ Species $^{a}$} & \multicolumn{15}{|c|}{$\%$ Similarity } \\
\hline & 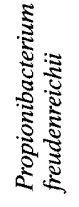 & 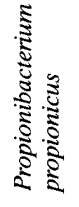 & 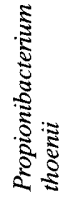 & 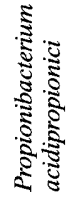 & 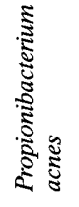 & 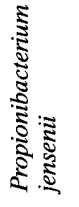 & 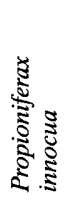 & 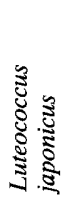 & 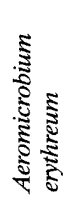 & 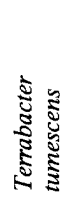 & 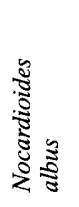 & 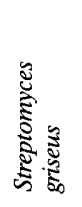 & 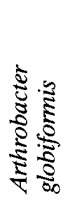 & 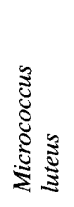 & 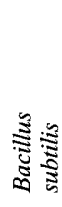 \\
\hline $\begin{array}{l}\text { Propionibacterium } \\
\text { cyclohexanicum }\end{array}$ & 97.1 & 95.7 & 95.0 & 94.9 & 94.9 & 94.9 & 94.3 & 93.4 & 92.4 & 91.9 & 91.8 & 91.3 & 90.9 & 90.6 & 81.5 \\
\hline $\begin{array}{l}\text { Propionibacterium } \\
\text { freudenreichii }\end{array}$ & & 95.8 & 95.4 & 95.5 & 95.3 & 95.3 & 94.2 & 92.7 & 92.9 & 91.2 & 91.8 & 90.9 & 91.2 & 90.9 & 82.0 \\
\hline $\begin{array}{l}\text { Propionibacterium } \\
\text { propionicus }\end{array}$ & & & 95.7 & 95.8 & 95.5 & 95.5 & 94.8 & 93.9 & 93.3 & 92.7 & 93.2 & 91.8 & 91.7 & 91.1 & 82.5 \\
\hline $\begin{array}{l}\text { Propionibacterium } \\
\text { thoenii }\end{array}$ & & & & 98.3 & 96.0 & 98.6 & 93.3 & 92.5 & 93.3 & 92.4 & 91.8 & 91.3 & 91.8 & 91.8 & 82.5 \\
\hline $\begin{array}{l}\text { Propionibacterium } \\
\text { acidipropionici }\end{array}$ & & & & & 96.3 & 98.0 & 93.0 & 91.3 & 92.9 & 91.6 & 91.6 & 90.7 & 91.2 & 91.2 & 83.0 \\
\hline $\begin{array}{l}\text { Propionibacterium } \\
\text { acnes }\end{array}$ & & & & & & 96.0 & 91.8 & 91.8 & 91.1 & 89.9 & 90.6 & 89.3 & 89.4 & 89.4 & 81.4 \\
\hline $\begin{array}{l}\text { Propionibacterium } \\
\text { jensenii }\end{array}$ & & & & & & & 93.2 & 91.9 & 92.8 & 91.7 & 91.3 & 90.8 & 90.9 & 91.1 & 82.2 \\
\hline $\begin{array}{l}\text { Propioniferax } \\
\text { innocua }\end{array}$ & & & & & & & & 93.8 & 94.4 & 92.2 & 94.2 & 92.1 & 91.2 & 91.2 & 83.1 \\
\hline $\begin{array}{l}\text { Luteococcus } \\
\text { japonicus }\end{array}$ & & & & & & & & & 92.4 & 91.8 & 92.4 & 90.6 & 90.6 & 89.9 & 82.4 \\
\hline $\begin{array}{l}\text { Aeromicrobium } \\
\text { erythreum }\end{array}$ & & & & & & & & & & 93.3 & 95.3 & 92.3 & 92.8 & 92.4 & 83.1 \\
\hline $\begin{array}{l}\text { Terrabacter } \\
\text { tumescens }\end{array}$ & & & & & & & & & & & 92.2 & 93.3 & 94.7 & 94.5 & 84.1 \\
\hline $\begin{array}{l}\text { Nocardioides } \\
\text { albus }\end{array}$ & & & & & & & & & & & & 91.2 & 91.6 & 91.2 & 82.2 \\
\hline $\begin{array}{l}\text { Streptomyces } \\
\text { griseus }\end{array}$ & & & & & & & & & & & & & 92.3 & 92.3 & 84.1 \\
\hline $\begin{array}{l}\text { Arthrobacter } \\
\text { globiformis }\end{array}$ & & & & & & & & & & & & & & 96.9 & 83.9 \\
\hline $\begin{array}{l}\text { Micrococcus } \\
\text { luteus }\end{array}$ & & & & & & & & & & & & & & & 84.2 \\
\hline
\end{tabular}

${ }^{a}$ The designations of the strains used are indicated in the text.

propionibacteria were around $95 \%$, and the levels of homology with other bacteria were lower. Figure 1 shows a phylogenetic tree based on the $16 \mathrm{~S}$ rRNA gene sequences of strain TA-12 ${ }^{\mathrm{T}}$, Propionibacterium species, and other related groups. Our strain was located closest to Propionibacterium freudenreichii.

\section{DISCUSSION}

Strain TA- $12^{\mathrm{T}}$ is a gram-positive, nonmotile, pleomorphic, rod-shaped bacterium. It produces propionic acid from glucose. MK-9 $\left(\mathrm{H}_{4}\right)$ is its respiratory quinone, and alanine, glu-

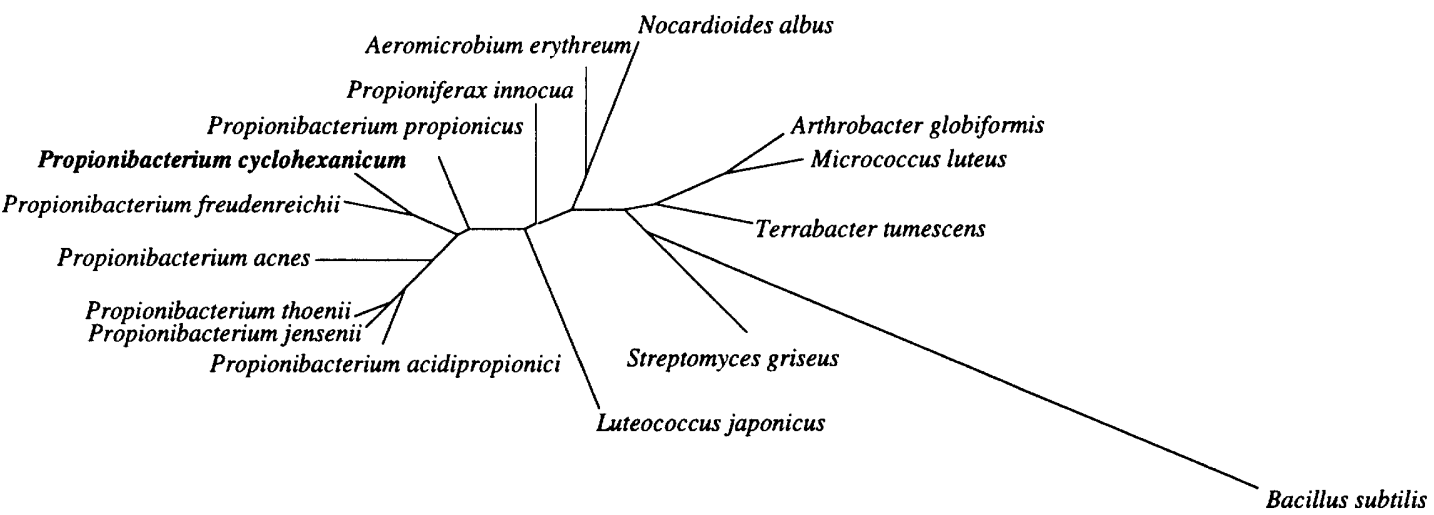

FIG. 1. Unrooted phylogenetic tree showing the positions of Propionibacterium cyclohexanicum TA-12 ${ }^{\mathrm{T}}$ and related species. The strains used are indicated in the 
tamic acid, and meso-diamoinopimelic acid are components of the cell wall. The whole-cell sugars are galactose, mannose, glucose, ribose, and rhamnose. The $\mathrm{G}+\mathrm{C}$ content of the DNA is $66.8 \mathrm{~mol} \%$. These morphological, biochemical, and chemotaxonomic features of strain TA- $12^{\mathrm{T}}$ are consistent with the characteristics of the genus Propionibacterium. Phylogenetically, strain TA-12 ${ }^{\mathrm{T}}$ is closely related to Propionibacterium freudenreichii DSM 20271, the type strain of the type species of the genus Propionibacterium. On the basis of its features and its phylogenetic location close to Propionibacterium freudenreichii DSM $20271^{\mathrm{T}}$, strain TA- $12^{\mathrm{T}}$ belongs to the genus Propionibacterium. Strain TA-12 ${ }^{\mathrm{T}}$ differs from previously described Propionibacterium species and other propionic acid-producing species by containing $\omega$-cyclohexyl fatty acid. $\omega$-Cyclohexyl fatty acid is a major cellular fatty acid of strain $\mathrm{TA}-12^{\mathrm{T}}$, and this fatty acid composition is unlike any previously reported cellular fatty acid composition of gram-positive, high-G+C-content bacteria except the Curtobacterium pusillum cellular fatty acid composition (28). Unlike strain TA-12 ${ }^{\mathrm{T}}, C$. pusillum contains MK-9 and D-ornithine (15). Strain TA- $12^{\mathrm{T}}$ produces lactic acid, which distinguishes it from all other species except Propionibacterium propionicus. These and other phenotypic features listed in Tables 1 and 2 clearly distinguish strain TA-12 from other propionibacteria. Thus, strain TA-12 ${ }^{\mathrm{T}}$ is distinct and represents a new species of the genus Propionibacterium.

Strain TA-12 ${ }^{\mathrm{T}}$ exhibited the highest level of 16S rRNA similarity $(97.1 \%$ ) with Propionibacterium freudenreichii; this value is such a low one that this bacterium does not hybridize with any bacteria at the species level (27). Strain TA-12 ${ }^{\mathrm{T}}$ is distinct both genetically and phenotypically.

The observed heat resistance of strain TA $-12^{\mathrm{T}}$ (it is able to survive at $90^{\circ} \mathrm{C}$ for $10 \mathrm{~min}$ ) is very high. Malik et al. (17) reported that Propionibacterium freudenreichii was the most heat resistant of the propionibacteria, but this organism was reported to be able to survive at $62.8^{\circ} \mathrm{C}$ for $30 \mathrm{~min}$. In our test, Propionibacterium freudenreichii could survive at $80^{\circ} \mathrm{C}$ for 10 min but could not survive at $90^{\circ} \mathrm{C}$ for $10 \mathrm{~min}$. Fruit juices are generally pasteurized at 90 to $95^{\circ} \mathrm{C}$ for $30 \mathrm{~s}$ to $1 \mathrm{~min}$ prior to packaging, and it is possible that heat-resistant bacteria such as strain TA $-12^{\mathrm{T}}$ can survive this treatment. Conversely, isolation of strain $\mathrm{TA}-12^{\mathrm{T}}$ from pasteurized and packaged orange juice substantiates its heat resistance. Despite the extraordinarily high resistance of strain $\mathrm{TA}-12^{\mathrm{T}}$ to heat, no spores similar to the endospores of members of the Bacillaceae and no specialized structures were observed by optical microscopy of sporestained cells or electron microscopy of thin sections of cells. This bacterium is a very unusual propionibacterium as it is able to grow at a low $\mathrm{pH}$ and is able to withstand heat treatment at $90^{\circ} \mathrm{C}$ for $10 \mathrm{~min}$.

Alicylic fatty acid is a rare cellular fatty acid. $\omega$-Cyclohexyl fatty acid has been found in Bacillus acidocaldarius (22) and Bacillus acidoterrestris (10), and $\omega$-cycloheptyl fatty acid has been obtained from Bacillus cycloheptanicus (21). These three species are all acidothermophilic and were classified as members of the genus Alicyclobacillus in 1992 (35). In 1981, Suzuki et al. reported that $C$. pusillum, a gram-positive, high-G+Ccontent bacterium, contains $\omega$-cyclohexyl fatty acid (29). This bacterium was isolated from oil brine (13) and is mesophilic and neutrophilic. Strain TA-12 ${ }^{\mathrm{T}}$ represents a third group of organisms which contain an alicylic fatty acid, and it is acidotolerant but not thermophilic. This shows that the presence of alicylic fatty acids in bacteria is not directly related to the apparent physiological properties of the organisms and is not related to what genus they belong to.

Description of Propionibacterium cyclohexanicum sp. nov. Propionibacterium cyclohexanicum (cy.clo.hex.a'ni.cum. Gr. n. kyklos, circle; Gr. n. hexa, six; M. L. masc. adj. cyclohexanicum, referring to $\omega$-cyclohexyl fatty acid, the characteristic cellular fatty acid of the organism). Cells are gram-positive, aerotolerant, nonmotile, non-acid-fast, non-spore-forming, pleomorphic rods. The cells are 1.5 to $3.0 \mu \mathrm{m}$ long and 1.1 to $1.6 \mu \mathrm{m}$ wide. Some cells are club shaped or bent. Colonies grown anaerobically on the surface of peptone-yeast extract-glucose agar for 3 days are circular, white to creamy, translucent, and 0.2 to 0.5 $\mathrm{mm}$ in diameter. The catalase, oxidase, and Voges-Proskauer tests are negative, and the methyl red test is positive. Urea and esculin are hydrolyzed. Gelatin and starch are not hydrolyzed. Nitrate is not reduced to nitrite, and indole is not produced. Glycerol, L-xylose, galactose, D-glucose, D-fructose, D-mannose, amygdalin, arbutin, esculin, salicin, cellobiose, maltose, lactose, saccharose, trehalose, melezitose, D-turanose, D-fucose, acetic acid, lactic acid, malic acid, citric acid, and succinic acid are assimilated. Erythritol, D-arabinose, ribose, adonitol, $\beta$-methylxyloside, L-sorbose, rhamnose, dulcitol, inositol, mannitol, sorbitol, $\alpha$-methylmannoside, $\alpha$-methylglucoside, $N$-acetylglucosamine, inulin, D-raffinose, starch, glycogen, xylitol, $\beta$-gentiobiose, D-lyxose, D-tagatose, L-fucose, L-arabitol, gluconate, 2-ketogluconate, and 5-ketogluconate are not assimilated. Lactic acid and propionic acid are the major end products of the glucose fermentation, and acetic acid is also detected. Grows at $\mathrm{pH} 3.2$ to 7.5 (optimum $\mathrm{pH}, 5.5$ to 6.5 ) and at 20 to $40^{\circ} \mathrm{C}$ (optimum temperature, $35^{\circ} \mathrm{C}$ ). The cells are heat resistant and survive at $90^{\circ} \mathrm{C}$ for $10 \mathrm{~min}$. The major menaquinone is MK-9 $\left(\mathrm{H}_{4}\right)$. $\omega$-Cyclohexyl undecanoic acid is the main cellular fatty acid. The isomer of diaminopimelic acid in the cell wall is meso-diaminopimelic acid. The $N$-glycolyl residue is not present. The cell wall amino acids are alanine, glutamic acid, and meso-diaminopimelic acid. The whole-cell sugars are galactose, glucose, mannose, ribose, and rhamnose. The $\mathrm{G}+\mathrm{C}$ content of the DNA is $66.8 \mathrm{~mol} \%$. The bacterium was isolated from pasteurized orange juice.

The type strain is TA-12 (= IAM $14535=$ NRIC 0247).

\section{ACKNOWLEDGMENTS}

We thank Akihiro Iwamatsu and Katsuya Okawa, Central Laboratories for Key Technology, KIRIN Brewery Co., Ltd., for the analysis of amino acids. We thank Tomonori Suzuki, Science University of Tokyo, for helpful suggestions regarding alignment of the sequence data and calculations of homology values.

\section{REFERENCES}

1. Bligh, E. G., and W. J. Dyer. 1959. A rapid method of total lipid extraction and purification. Can. J. Biochem. Physiol. 37:911-917.

2. Cerny, G., W. Hennlich, and K. Poralla. 1984. Spoilage of fruit juice by bacilli: isolation and characterization of the spoiling microorganism. Z. Lebensm. Unters. Forsch. 179:224-227.

3. Charfreitag, O., M. D. Collins, and E. Stackebrandt. 1988. Reclassification of Arachnia propionica as Propionibacterium propionicus comb. nov. Int. J. Syst. Bacteriol. 38:354-357.

4. Cohen, S. A., and D. P. Michaud. 1993. Synthesis of a fluorescent derivatizing reagent, 6-aminoquinolyl- $N$-hydroxysuccinimidyl carbamate, and its application for the analysis of hydrolysate amino acids via high-performance liquid chromatography. Anal. Biochem. 211:279-287.

5. Cowan, S. T., and K. J. Steel. 1965. Manual for the identification of medical bacteria. Cambridge University Press, London, United Kingdom.

6. Cummins, C. S., and J. L. Johnson. 1981. The genus Propionibacterium, p. 1894-1902. In M. P. Starr, H. Stolp, H. G. Trüper, A. Balows, and H. G. Schlegel (ed.), The procaryotes, vol. 1. Springer-Verlag, New York, N.Y.

7. Cummins, C. S., and J. L. Johnson. 1986. Genus Propionibacterium, p. 1346-1353. In P. H. A. Sneath, N. S. Mair, M. E. Sharpe, and J. G. Holt (ed.), Bergey's manual of systematic bacteriology, vol. 2. The Williams \& Wilkins Co., Baltimore, Md.

8. Deinhard, G., P. Blanz, K. Poralla, and E. Altan. 1987. Bacillus acidoterrestris sp. nov., a new thermotolerant acidophile isolated from different soils. Syst. Appl. Microbiol. 10:47-53.

9. Ehresmann, C., P. Stiegler, P. Fellner, and J. P. Ebel. 1972. The determination of the primary structure of the $16 \mathrm{~S}$ ribosomal RNA of Escherichia coli. Biochimie 54:901-967. 
10. Hippchen, B., A. Röll, and K. Poralla. 1981. Occurrence in soil of thermoacidophilic bacilli possessing $\omega$-cyclohexane fatty acids and hopanoids. Arch. Microbiol. 129:53-55.

11. Hucker, G. J., and H. J. Conn. 1923. Methods of Gram staining. Tech. Bull N. Y. State Agric. Exp. Stn. 93:3-37.

12. Hughes, R. C. 1965 . The isolation of structural components present in the cell wall of Bacillus licheniformis N.C.T.C. 6346. Biochem. J. 96:700-709.

13. Iizuka, H., and K. Komagata. 1965. Determination of Brevibacterium, Arthrobacter, Micrococcus, Sarcina, Alcaligenes, and Achromobacter isolated from oil-brines in Japan. J. Gen. Appl. Microbiol. 11:1-14.

14. Jones, D., and M. D. Collins. 1986. Irregular, nonsporing gram-positive rods, p. 1261-1266. In P. H. A. Sneath, N. S. Mair, M. E. Sharpe, and J. G. Holt (ed.), Bergey's manual of systematic bacteriology, vol. 2. The Williams \& Wilkins Co., Baltimore, Md.

15. Komagata, K., and K. Suzuki. 1986. Genus Curtobacterium, p. 1313-1317. In P. H. A. Sneath, N. S. Mair, M. E. Sharpe, and J. G. Holt (ed.), Bergey's manual of systematic bacteriology, vol. 2. The Williams \& Wilkins Co. Baltimore, Md

16. Kooiman, W. J. 1969. The screw-cap tube technique: a new and accurate technique for the determination of the wet heat resistance of bacteria spores, p. 87-92. In G. W. Gould and A. Hurst (ed.), The bacterial spore Academic Press, London, United Kingdom.

17. Malik, A. C., G. W. Reinbold, and E. R. Vedamuthu. 1968. An evaluation of the taxonomy of Propionibacterium. Can. J. Microbiol. 14:1185-1191.

18. Meade, H. M., S. R. Long, C. B. Ruvkun, S. E. Brown, and F. M. Ausubel 1982. Physical and genetic characterization of symbiotic and auxotrophic mutants of Rhizobium meliloti induced by transposon Tn 5 mutagenesis. J. Bacteriol. 149:114-122.

19. Metcalfe, L. D., A. A. Schmitz, and J. R. Pelka. 1996. Rapid preparation of fatty acid esters from lipids for gas chromatographic analysis. Anal. Chem. 38:514-515.

20. Pitcher, D. G., and M. D. Collins. 1991. Phylogenetic analysis of some LL-diaminopimelic acid-containing coryneform bacteria from human skin: description of Propionibacterium innocuum sp. nov. FEMS Microbiol. Lett. 84:295-300.

21. Poralla, K., and W. A. König. 1983. The occurrence of $\omega$-cycloheptane fatty acids in a thermo-acidophilic bacillus. FEMS Microbiol. Lett. 16:303-306.

22. Rosa, M. D., A. Gambacorta, L. Minale, and J. D. Bu'Lock. 1971. Cyclohex ane fatty acids from a thermophilic bacterium. Chem. Comm. 1334-1334.

23. Saiki, R. K., D. H. Gelfand, S. Stoffe, S. J. Scharf, R. Higuchi, G. T. Horn,
K. B. Mullis, and A. Erlich. 1988. Primer-directed enzymatic amplification of DNA with a thermostable DNA polymerase. Science 239:487-491.

24. Schaal, K. P. 1986. The genus Arachnia, p. 1332-1342. In P. H. A. Sneath, N. S. Mair, M. E. Sharpe, and J. G. Holt (ed.), Bergey's manual of systematic bacteriology, vol. 2. The Williams \& Wilkins Co., Baltimore, Md.

25. Schaeffer, A. B., and M. D. Fulton. 1933. A simplified method of staining endospores. Science 77:194.

26. Splittstoesser, D. F., J. J. Churey, and C. Y. Lee. 1994. Growth characteristics of aciduric sporeforming bacilli isolated from fruit juices. J. Food Prot. 57:1080-1083.

27. Stackebrandt, E., and B. M. Goebel. 1994. Taxonomic note: a place for DNA-DNA reassociation and 16S rRNA sequence analysis in the present species definition in bacteriology. Int. J. Syst. Bacteriol. 44:846-849.

28. Staneck, J. L., and G. D. Roberts. 1974. Simplified approach to identification of aerobic actinomycetes by thin-layer chromatography. Appl. Microbiol. 28:226-231.

29. Suzuki, K., K. Saito, A. Kawaguchi, S. Okuda, and K. Komagata. 1981 Occurrence of $\omega$-cyclohexyl fatty acids in Curtobacterium pusillum strains. J. Gen. Appl. Microbiol. 27:261-266.

30. Tamaoka, J., and K. Komagata. 1984. Determination of DNA base composition by reversed-phase high-performance liquid chromatography. FEMS Microbiol. Lett. 25:125-128.

31. Tamaoka, J., Y. Katayama-Fujimura, and H. Kuraishi. 1983. Analysis of bacterial menaquinone mixtures by high performance liquid chromatography. J. Appl. Bacteriol. 54:31-36.

32. Thompson, J. D., D. G. Higgins, and T. J. Gibson. 1994. CLUSTAL W: improving the sensitivity of progressive multiple sequence alignment through sequence weighting, positions-specific gap penalties and weight matrix choice. Nucleic Acids Res. 22:4673-4680.

33. Treisman, R. 1989. Purification of plasmid DNA, p.1.40-1.41. In J. Sambrook, E. F. Fritsch, and T. Maniatis (ed.), Molecular cloning, 2nd ed. Cold Spring Harbor Laboratory Press, Cold Spring Harbor, N.Y.

34. Uchida, K., and K. Aida. 1984. An improved method for the glycolate test for simple identification of the acyl type of bacterial cell walls. J. Gen. Appl Microbiol. 30:131-134.

35. Wisotzkey, J. D., P. Jurtshuk, Jr., G. E. Fox, G. Deinhard, and K. Poralla 1992. Comparative sequence analyses on the 16S rRNA (rDNA) of Bacillus acidocaldarius, Bacillus acidoterrestris, and Bacillus cycloheptanicus and proposal for creation of a new genus, Alicyclobacillus gen. nov. Int. J. Syst. Bacteriol. 42:263-269. 